\title{
Projeto colaborativo em ambientes digitais de atividades de aprendizagem e avaliação para aquisição de competências em informação e documentação
}

\author{
Joint project in digital learning environments and \\ an evaluation in obtaining information \\ and documentation skills
}

\author{
Maria Del Carmen AGUSTÍN-LACRUZ' \\ Raquel GÓMEZ-DÍAZ² \\ Mariângela Spotti Lopes FUJITA ${ }^{3}$
}

\section{Resumo}

A busca de projeção internacional e a necessidade de formar redes formais ou informais de cooperação acadêmica são algumas das características mais comuns da formação atual, oferecidas por várias universidades. Cientes dessa dificuldade, assim como da conveniência da criação de sinergias docentes e do enriquecimento das redes de colaboração acadêmica entre as universidades, é apresentada no contexto de ensino de Informação e Documentação, uma experiência de colaboração interuniversitária para o projeto conjunto de atividades de aprendizagem e avaliação por competências, desenvolvido por docentes das Universidades Públicas de Zaragoza e Salamanca (Espanha) e da Universidade Estadual Paulista Júlio de Mesquita Filho (São Paulo, Brasil). A experiência é desenvolvida por meio de modelos de registros que facilitem a estruturação de atividades de aprendizagem que visam à aquisição de competências comuns. Cada atividade proposta e desenvolvida é registrada em uma planilha que reúne a informação, organizada em diversos campos como: descrição, competências, objetivos, resultados de aprendizagem previstos, ferramentas, recursos e materiais necessários, critérios de avaliação, entre outros aspectos, de maneira que o aluno possa ver o que deve fazer, como fazer e qual a utilidade. Essa forma de conceber as atividades de aprendizagem baseadas em competências é possível, em ambientes acadêmicos geograficamente tão distantes, pelo uso das Tecnologias de Informação e Comunicação, que permitem tanto a colaboração à distância entre os professores quanto o acesso aos materiais oferecidos nas plataformas de cada uma das universidades.

Palavras-chave: Aprendizagem baseada em competências. Espaço europeu de educação superior. Projeto de atividades de aprendizagem. Tecnologia de informação e comunicação.

\begin{abstract}
The search for international impact and the need to create formal or informal networks of academic cooperation are some of the most common features of the current training offered by many universities. A ware of this difficulty and the desirability of creating synergies to enrich teaching and academic collaboration networks between universities, an experience is presented, in the context of the teaching of Information and Documentation, of inter-university collaboration for the joint design of learning activities and skills assessment, developed by teachers in the public universities of Zaragoza and Salamanca (Spain) and the São Paulo Universidade Estadual Paulista Júlio de Mesquita Filho

\footnotetext{
1 Universidad de Zaragoza, Facultad de Filosofía y Letras, Centro de la Documentación e História de la Ciencia. Zaragoza, España.

2 Universidad de Salamanca, Facultad de Traducción y Documentación, Departamento de Biblioteconomía y Documentación. Salamanca, España.

3 Universidade Estadual Paulista Júlio de Mesquita Filho, Faculdade de Filosofia e Ciências, Departamento de Ciência da Informação. Campus de Marília, Av. Higyno Muzzi Filho, 737, 17525-900, Marília, SP, Brasil. Correspondência para/Correspondence to: M.S.L. FUJITA. E-mail: <fujita@marilia.unesp.br>.

Recebido em 10/4/2011 e aceito para publicação em 21/6/2011.
} 
(São Paulo, Brazil). The experience is developed using models that facilitate the structuring of learning activities aimed at the acquisition of common skills. Each activity that is proposed and developed is recorded in a spreadsheet which collects the information arranged in various fields such as: description, skills, objectives, expected learning outcomes, tools, required resources and materials, evaluation criteria, amongst others, so that the student can see what he is asked to do, how to do it and how useful it will be. This way of designing skills-based learning activities is possible, in geographically diverse academic settings through the use of Information Technology and Communication, enabling both remote cooperation between teachers and also materials offered on the platforms of each of the universities.

Keywords: Competence based learning. European higher education area. Design of learning activities. Information technology and communication.

\section{Introdução}

Oferta de formação e currículo baseada em competências

Um dos traços mais característicos do ensino superior nas sociedades contemporâneas é a preocupação em fornecer aos alunos uma qualificação de alto nível relacionada ao desempenho profissional.

Isso explica por que, no âmbito do ensino superior atual, se ampliou o discurso articulado sobre a educação ao longo da vida, empregabilidade e versatilidade profissional. Nesse contexto, é relevante a ideia de que a aprendizagem deve ser orientada para a aquisição de habilidades ou competências e a formação para o desempenho dos processos. Em paralelo, tem emergido como um novo vetor ou eixo de interesse educativo o conceito de"competência" (Alonso et al., 2009).

Essa ideia surgiu na década de sessenta do século passado, nos Estados Unidos e Grã-Bretanha (Argüelles; Gonczi, 2001), relacionada a uma concepção de ensino superior que propunha acompanhar a formação universitária às necessidades da economia - fundamentalmente industrial - americana, exercendo um papel maior do que a aquisição de conhecimentos teóricos, em competências para o exercício de uma profissão ou para a realização de exercícios profissionais específicos.

Por essa razão, uma das definições mais gerais identifica competência como saber fazer em um contexto, em que se entende que saber fazer reflete a capacidade de executar uma função ou atividade. As competências transcendem o"conhecer"e o"fazer" clássicos. A formação com base nelas (Figura 1) integra conhecimentos, aptidões, valores e habilidades que permitem alcançar o desempenho profissional em situações determinadas.

O planejamento por competências facilita a formação integral dos alunos, exigindo: a) uma relação estreita entre teoria e prática, b) uma atitude crítica para interagir com situações concretas e, c) capacidade de

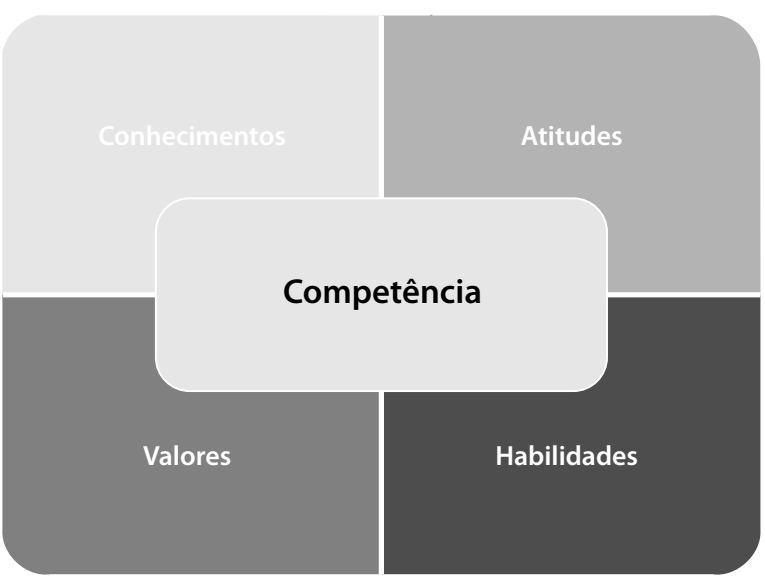

Figura 1. Conceito de competência.

tomar decisões e resolver problemas (Agustín-Lacruz, 2008; Sánchez Casabón; Agustín-Lacruz, 2009).

O surgimento do enfoque em competências permitiu superar a orientação tradicional baseada nos conteúdos teóricos e concebeu o currículo a partir de novas perspectivas, indo além "das estruturas e da lógica histórica das disciplinas" e exigindo um enfoque "inter-, co- e transdisciplinar" dentro de cada uma delas, como apontado por Posada Álvarez (2005, p.23). A formação baseada na aquisição de competências exige a integração do corpus de conhecimentos, das habilidades, das atividades práticas e dos valores nas disciplinas clássicas.

No entanto, em paralelo, também surgiram críticas que chamam a atenção sobre a origem empresarial e relacionada com o mundo dos negócios desse modelo educativo. Como consta do Relatório da OCDE, Habilidades e competências do século XXI para os alunos do novo milênio nos países da OCDE (Organización para la Cooperación y el Desarrollo Económicos, 2010, p.4):

Ao invés de colocar a ênfase no desenvolvimento harmonioso de todas as capacidades humanas, o discurso sobre as competências reforça a importância das competências relacionadas ao trabalho. Além disso, alguns argumentam que, 
tal e como estão definidas, nem todos os alunos alcançarão as habilidades do século XXI, porque, primeiro nem todos os jovens de hoje serão [os] trabalhadores altamente qualificados de amanhã nem mesmo em países desenvolvidos e, segundo, porque essa retórica esquece as necessidades da grande maioria dos países do mundo.

Essas críticas defendem que, embora o enfoque das competências seja muito valioso para orientar o ensino e a aprendizagem em sala de aula, a retórica em torno desse conceito está perto de uma educação cujo único objetivo é preparar trabalhadores qualificados para economias do conhecimento e, até mesmo, para empresas concretas.

\section{O ponto de partida}

A implantação, em um grande número de países, do modelo organizacional proposto no Espaço Europeu de Educação Superior (EEES) abriu as portas para um sistema educacional organizado em torno de competências (Tejada Artigas, 2009).

Nesse sentido, para fortalecer sua posição estratégica como serviço público, a maioria das universidades participa de redes de cooperação acadêmica de ensino e pesquisa, com caráter supraterritorial e alcance internacional. Em um contexto no qual as tecnologias de informação e redes de comunicação oferecem oportunidades de interconexão inimagináveis há algumas décadas (García Orozco, 2010), e com o objetivo de reforçar as sinergias educativas e de enriquecer as redes de colaboração acadêmica entre as universidades (Figura 2), é apresentada neste artigo uma experiência de colaboração interuniversitária para a concepção conjunta de atividades de aprendizagem e avaliação por competências, proposta como meta final para promover melhorias na qualidade do aprendizado dos alunos envolvidos no Projeto (Gómez Díazetal., 2009)

O trabalho tem seu ponto de partida em três pilares: a) uma visão comum do papel do ensino superior; b) os modelos de desempenho docente e afins; c) os modos de ação semelhantes.

1. O Projeto é realizado em conjunto pelos professores e alunos das universidades públicas espanholas de Zaragoza e Salamanca e da Universidade Estadual Paulista Júlio de Mesquita Filho (São Paulo, Brasil), no

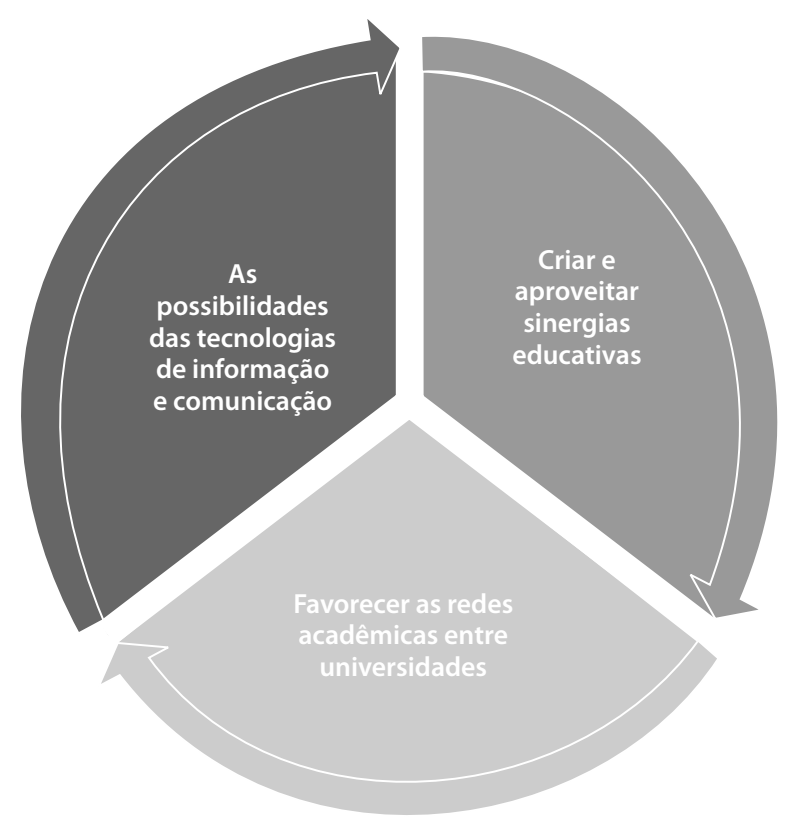

Figura 2. Contexto do Projeto.

âmbito da disciplina de Informação e Documentação (Figura 3).

Os três professores integrantes da equipe de trabalho desenvolveram sua atividade de ensino e pesquisa no âmbito da análise, representação e recuperação da informação em ambientes documentários. Esse campo de trabalho está confinado dentro dos limites definidos pelas competências específicas de formação disciplinar em Informação e Documentação E07, E08 e E09, estabelecidas no seu Livro Branco (Agencia Nacional de Evaluación de La Calidad y Acreditación, 2004, p.59), (Quadro 1).

Esta experiência se propõe a refletir de forma critica sobre o trabalho dos docentes envolvidos no Projeto, em duas direções (Figura 4): (1) analisando qual deve ser a escolha adequada dos conteúdos teóricos, planejamento de atividades de aprendizagem e avaliações de controle da aprendizagem. A variedade de contextos educativos em que tal reflexão tem lugar, assim como a diversidade institucional e geográfica, enriquece o valor da reflexão; (2) oferecendo aos alunos uma ferramenta que Ihes permita conhecer e realizar a sequência detalhada do marco teórico disciplinar, o planejamento dos conteúdos, as atividades de aprendizagem que lhes permitam adquirir e estabelecer as competências, a tipologia e critérios dos testes de avaliação etc. 


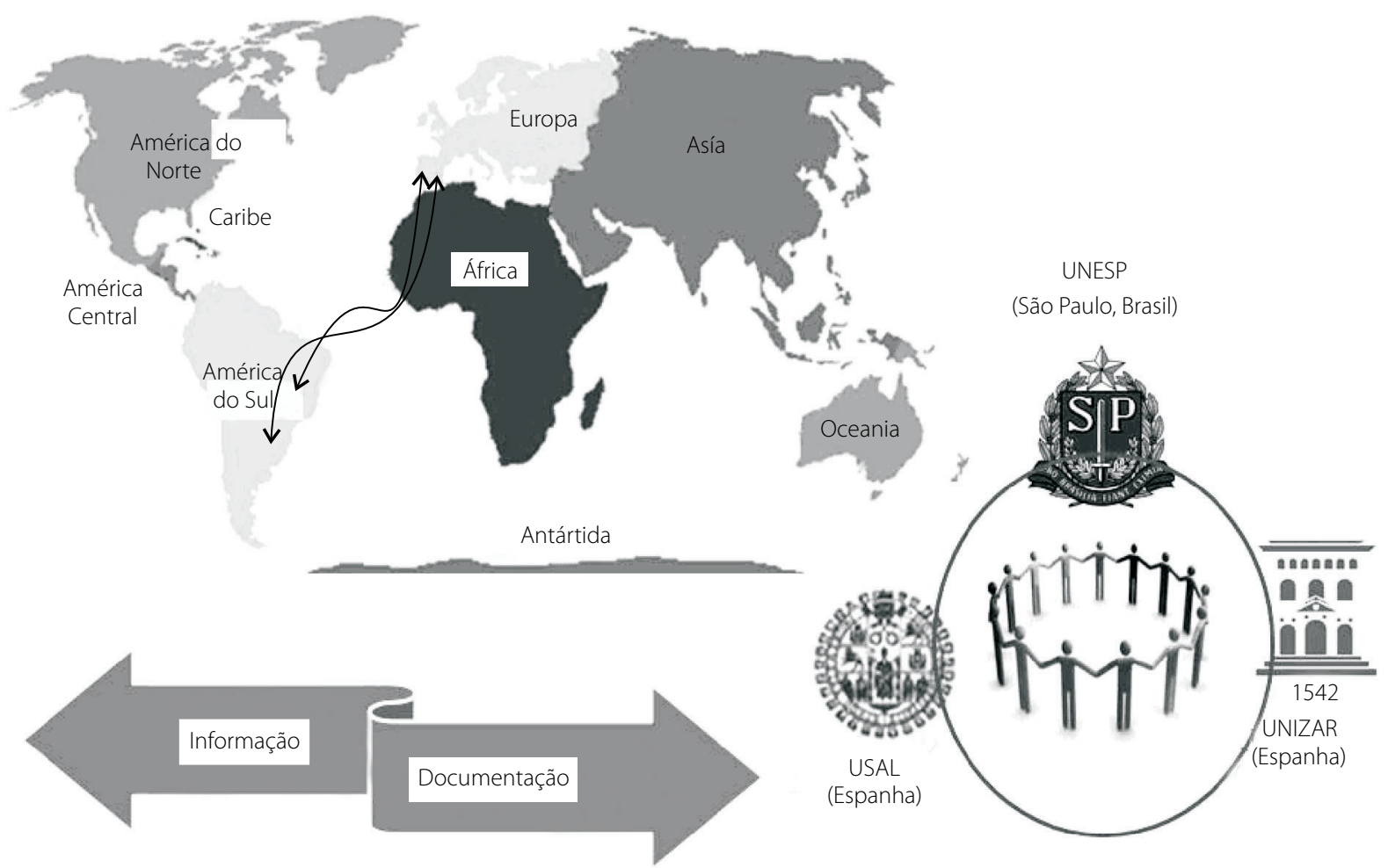

Figura 3. Âmbito disciplinar, institucional e geográfico do Projeto.

Nota: UNESP: Universidade Estadual Paulista Júlio de Mesquita Filho; USAL: Universidad de Salamanca; UNIZAR: Universidad de Zaragoza.

Quadro 1. Competências específicas de informação e documentação.

\begin{tabular}{lll}
\hline Competência & Denominação & Descrição \\
\hline E07 & $\begin{array}{l}\text { Análise e Representação da In- } \\
\text { formação }\end{array}$ & $\begin{array}{l}\text { Identificar e representar em linguagem documentária adaptada ou em outro siste- } \\
\text { ma simbólico o conteúdo semântico de um documento, de uma coleção de do- } \\
\text { cumentos ou de um arquivo } \\
\text { E08 }\end{array}$ \\
$\begin{array}{ll}\text { Organização e Armazenamento } \\
\text { da Informação }\end{array}$ & $\begin{array}{l}\text { Organizar e estruturar os dados relativos à descrição de documentos e coleções de } \\
\text { documentos em qualquer suporte, criar e explorar as ferramentas de acesso a da- } \\
\text { dos, documentos ou referências }\end{array}$ \\
Busca e Recuperação da Infor- & $\begin{array}{l}\text { Buscar e recuperar a informação por métodos que possam responder às expecta- } \\
\text { tivas dos usuários em ótimas condições de custo e tempo. }\end{array}$ \\
\hline
\end{tabular}

\section{Método de trabalho}

O Projeto é desenvolvido em ambiente digital, mediante a elaboração de fichas-guia para provas documentárias, que facilitam a metacognição sobre os processos de ensino-aprendizagem e registram seu acompanhamento e resultados.

As ferramentas baseadas nas Tecnologias de Comunicação e Informação (TIC) permeiam todo o método de trabalho: (a) permitem o desenvolvimento, distribuição e acompanhamento de todo o processo de ensino e aprendizagem de forma dinâmica e flexível; (b) constituem o meio de comunicação entre professores e alunos; (c) facilitam o trabalho colaborativo a partir de locais distantes, em tempo real; (d) viabilizam que todas as fichasguia sejam depositadas nas plataformas docentes digitais respectivas, assim como todos os recursos e materiais didáticos necessários para o desenvolvimento das atividades propostas. 


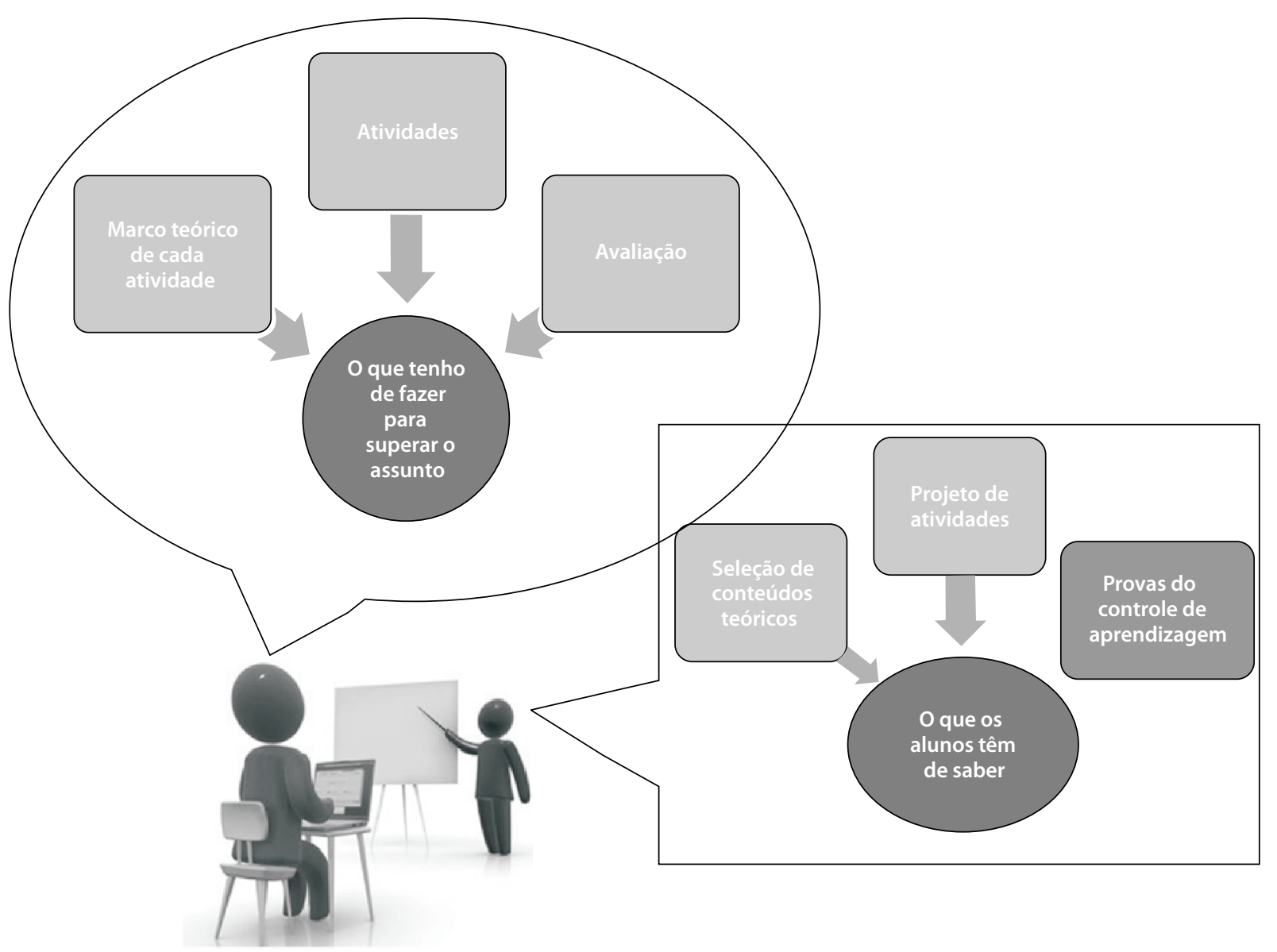

Figura 4. Objetivos de alunos e professores.

\section{Materiais}

O trabalho é desenvolvido mediante modelos de registros em que são coletados, de forma sistemática, os diversos aspectos que facilitam a estruturação de atividades de aprendizagem que visam à aquisição de competências comuns.

Cada atividade proposta e desenvolvida é registrada numa planilha, que reúne a informação organizada em diversos campos, tais como: a descrição, as competências, os objetivos, os resultados de aprendizagem previstos, as ferramentas, recursos e materiais necessários, os critérios de avaliação, entre outros aspectos, de modo que o aluno possa ver o que deve fazer, como fazer e qual a utilidade disso.

Os pontos relativos a cada atividade de aprendizagem são: (a) objetivos; (b) competências que se trabaIham; (c) resultados previstos de aprendizagem, expressos a partir da perspectiva do aluno; (d) marco teórico da atividade; (e) seleção de leituras que compõem o marco teórico; (f) ferramentas, materiais e recursos didáticos necessários: aplicações informáticas, base de dados, tesauro, repositório etc.; (g) desenvolvimento da atividade: procedimento a ser seguido pelo aluno e proposição de exemplos como orientação; (h) procedimentos, critérios e recomendações para as atividades de avaliação.

Como o modelo é baseado no projeto de aprendizagem por competências, é considerado adequado para expressá-las, como aparecem no Libro Blanco del título de Grado en Información y Documentación (Agencia Nacional de la Evaluación de la Calidad y Acreditación, 2004) ou, se for o caso, no Euroreferencial en Información y Documentación (European Council of Information Associations, 2004; Kajberg; Lørring, 2005).

Assim mesmo, pode ser interessante recorrer ao nível de especificidade com que se trabalha cada competência. Os níveis estabelecidos são: 
1) Sensibilização: se o aluno conhece a existência dos elementos de uma determinada função e manipula o vocabulário para identificar os problemas;

2) Conhecimento das práticas: na realidade, trata-se do primeiro nível profissional, já que o sujeito pode lidar com ferramentas básicas e é capaz de realizar certas tarefas técnicas;

3) Domínio de ferramentas: se o aluno já controla as diferentes técnicas e, portanto, é capaz de desenvolver novas ferramentas;

4) Domínio metodológico: capacidade de planejar estrategicamente e desenvolver novas ferramentas e produtos.

\section{Conclusão}

Este trabalho de elaboração de atividades de aprendizagem e registro dos resultados, baseado em competências e desenvolvido em ambientes marcados pelas TIC, proporciona benefícios para todos os intervenientes no processo educativo.

a) Para os alunos: permite identificar o que fazer e como devem fazer para adquirir uma competência; facilita a elaboração sistemática e estruturada dos registros das atividades de aprendizagem (portfólio discente); promove a aprendizagem significativa e a meta-cognição sobre o próprio processo de aprendizagem.

b) Para os professores: facilita o acompanhamento do processo de formação e do nível de aquisição de cada competição; permite comparar a aprendizagem entre os diferentes alunos; facilita o trabalho colaborativo com os colegas de outras instituições.

c) Para as instituições acadêmicas: promove a projeção da universidade; reforça as redes de colaboração entre instituições pertencentes a âmbitos geográficos, administrativos etc. diferentes.

\section{Referências}

AGENCIA NACIONAL DE AVALUACIÓN DE LA CALIDAD Y ACREDITACIÓN. Título de grado en información y documentación. Madrid: Agencia Nacional de Evaluación de la Calidad y Acreditación, 2004. Disponible en: <http:// www.aneca.es/media/150424/libroblanco_jun05_ documentacion.pdf>. Acceso: 26 nov. 2010.

AGUSTíN-LACRUZ, M.C. Diseño curricular y guías docentes ECTS: desde la diplomatura de biblioteconomía y documentación hasta el grado en información y documentación. Zaragoza: Prensas de la Universidad de Zaragoza, 2008.

ALONSO, L.E.; FERNÁNDEZ RODRÍGUEZ, S.J.; NYSSEN, J. M. EI debate sobre las competencias: una investigación cualitativa en torno a la educación superior y el mercado de trabajo en España. Madrid: Agencia Nacional de Evaluación de la Calidad y Acreditación, 2009. Disponible en: <http://www.aneca.es/ media/148145/publi_competencias_090303.pdf>. Acceso en: 27 nov. 2010.

ARGÜELLES, A.; GONCZI, A. Educación y capacitación basada en normas de competencias: una perspectiva internacional. México: Limusa, 2001.

EUROPEAN COUNCIL OF INFORMATION ASSOCIATIONS. Euroreferencialen Informacióny Documentación. Madrid:SEDIC, 2004. 2v.

GARCÍA OROZCO, F.J. Gestión de la información yel conocimiento: observatorio para la educación en ambientes virtuales. Guadalajara, Jalisco: Universidad de Guadalajara, 2010.

GÓMEZ-DÍAZ, R.; AGUSTÍN LACRUZ, M.C.; IZQUIERDO ALONSO, M. Innovación e investigación educativa en
Información y documentación en la universidad española entre 1998 y 2008. In: MANUEL BORGES, M.; SANZ CASADO, E. (Ed.). A ciência da informação criadora de conhecimiento. Coimbra: Universidade de Coimbra, 2009. v.2. p.339-350.

KAJBERG, L.; LØRRING, L. European curriculum reflections on library and information science education. Copenhage: The Royal School of Library and Information Science, 2005. Available from: <http://www.kf.vu.lt/site_files_doc/LIS_ Bologna.pdf>. Cited: 3 Nov. 2010.

ORGANIZACIÓN PARA LA COOPERACIÓN Y EL DESARROLLO ECONÓMICOS. Habilidades y competencias del siglo XXI para los aprendices del nuevo milenio en los países de la OCDE. [s.I.]: OCDE, 2010. Disponible en: <http://recursostic.educacion.es/ blogs/europa/media/blogs/europa/informes/Habilidades _y_competencias_siglo21_OCDE.pdf>. Acceso: 28 nov. 2010.

POSADA ÁLVAREZ, R. Formación superior basada en competencias: interdisciplinariedad y trabajo autónomo del estudiante. Revista Iberoamericana de Educación, n. 34, p.6, 2005. Disponible en: <http://www.campus-oei.org/revista/ edu_sup22.htm>. Acceso: 23 nov. 2010.

SÁNCHEZ CASABÓN, A.I.; AGUSTÍN-LACRUZ, M.C. Grado de información y documentación: coordinación curricular, diseño y redacción de guías docentes. Zaragoza: Prensas de la Universidad de Zaragoza, 2009.

TEJADA ARTIGAS, C.M. Competencias profesionales y formación universitaria en información y documentación. Educación y Biblioteca, v.21, n.170, p.83-87, 2009. 